\title{
Stability of stationary solutions to 2D-Navier-Stokes models with delays
}

\author{
Tomás Caraballo and Xiaoying Han
}

\author{
Communicated by Yuncheng You, received May 11, 2014. \\ Dedicated to Peter Kloeden on occasion of his 65th birthday.
}

\begin{abstract}
In this paper we establish some sufficient conditions for the exponential stability of the stationary solution to a two-dimensional Navier-Stokes model with delay in the forcing term. We are able to cover several situation in a single formulation by using functional formulation for the delay. In particular, our results improve some existing ones in the literature, which were only proved for variable delay.
\end{abstract}

\section{Contents}

1. Introduction

2. Preliminaries

3. Existence, uniqueness and regularity of solutions

4. Existence and exponential stability of stationary solutions

\section{Introduction}

Navier-Stokes equations have been receiving much attention over the last decades because of their important contributions to understanding fluids motion and turbulence (see [7], [10], [15], amongst others). For Navier-Stokes models containing some hereditary features in the forcing term, an investigation was initiated in 2001 by Caraballo and Real $[\mathbf{3}, \mathbf{4}, \mathbf{5}]$. After establishing the existence and eventual

1991 Mathematics Subject Classification. Primary 35R10, 35B40; Secondary 47H20, $58 \mathrm{~F} 39,73 \mathrm{~K} 70$.

Key words and phrases. Navier-Stokes equations, stability, variable delay, distributed delay, measurable delay.

Partly supported by Ministerio de Economía y Competitividad (Spain) and FEDER (European Community), project MTM2011-22411, and by Junta de Andalucía (Spain) under the Proyecto de Excelencia P12-FQM-1492. 
uniqueness of solutions in [3], some first results on the asymptotic behavior of solutions were established in $[\mathbf{4}, \mathbf{5}]$, the latter concerning the existence of pullback attractors.

Studies on the stability of the stationary solutions to Navier-Stokes models have been carried out in several papers, including the previously mentioned one by Caraballo and Real. Nevertheless for most cases they refer to only the situation in which the delay is of variable delay nature (see, for instance, Taniguchi [13], Garrido-Atienza and Marín-Rubio [9], Planas and Hernández [12]). To our best knowledge, only in [4] the stability was proved by using the Razumikhin approach, which allows more general delays but requires a rather strong condition of the continuity of operators with respect to time.

It is worth mentioning that some results ensuring the existence of absorbing sets are proved in [13], but the stability of stationary solutions is only analyzed for continuously differentiable variable delays when zero is the stationary solution. A stochastic perturbation of a two dimensional Navier-Stokes problem along with its deterministic counterpart were studied in $[\mathbf{6}]$. The reader is referred to the recent survey by Caraballo and Han [2] for a more detailed description of the various methods used to study stability of stationary solutions of 2D Navier-Stokes models with variable delay forcing terms.

In this paper, we will provide several results on the stability of stationary solutions to Navier-Stokes models with a functional formulation of the memory term, which allows more general types of delay, e.g., distributed delay. Our results improve some existing ones in the literature, in particular some results from Caraballo and Real [4], Taniguchi $[\mathbf{1 3}]$ and Chen $[\mathbf{6}]$.

The rest of the paper is organized as follows. In Section 2 we will set up the Navier-Stokes problem and provide some preliminary results on the 2D NavierStokes model with delay. Section 3 will be concerned with the existence and eventual uniqueness of solutions to our model, with an emphasis on stationary solutions. Then, the local asymptotic behavior will be analyzed in Section 4, and our results will be compared with those appeared in the literature.

\section{Preliminaries}

We start from a general formulation of the model used in our analysis.

Let $\Omega \subset \mathbb{R}^{2}$ be an open and bounded set with regular boundary $\Gamma$. Given $T>0$, we consider the following functional Navier-Stokes problem (for further details and notations see Lions $[\mathbf{1 1}]$ and Temam $[\mathbf{1 4}])$ :

$$
\begin{aligned}
\frac{\partial u}{\partial t} & =\nu \Delta u-\sum_{i=1}^{2} u_{i} \frac{\partial u}{\partial x_{i}}+f-\nabla p+g\left(t, u_{t}\right) \quad \text { in }(0, T) \times \Omega, \\
\operatorname{div} u & =0 \quad \text { in }(0, T) \times \Omega, \\
u & =0 \text { on }(0, T) \times \Gamma, \\
u(0, x) & =u_{0}(x), \quad x \in \Omega, \\
u(t, x) & =\phi(t, x), \quad x \in \Omega, t \in(-h, 0),
\end{aligned}
$$


where $u$ is the velocity field of the fluid, $\nu>0$ is the kinematic viscosity, $p$ is the pressure, $f$ is a nondelayed external force field, $g$ is another external force containing hereditary characteristics, $u_{0}$ is the initial velocity field and $\phi$ the initial datum in the interval of time $(-h, 0)$ with $h>0$ fixed.

Define the following abstract spaces:

- $\mathcal{V}=\left\{u \in\left(C_{0}^{\infty}(\Omega)\right)^{2}: \operatorname{div} u=0\right\}$.

- $H=$ the closure of $\mathcal{V}$ in $\left(L^{2}(\Omega)\right)^{2}$ with the norm $|\cdot|$, and inner product $(\cdot, \cdot)$ where for $u, v \in\left(L^{2}(\Omega)\right)^{2}$,

$$
(u, v)=\sum_{j=1}^{2} \int_{\Omega} u_{j}(x) v_{j}(x) d x .
$$

- $V=$ the closure of $\mathcal{V}$ in $\left(H_{0}^{1}(\Omega)\right)^{2}$ with the norm $\|\cdot\|$, and associated scalar product $((\cdot, \cdot))$, where for $u, v \in\left(H_{0}^{1}(\Omega)\right)^{2}$,

$$
((u, v))=\sum_{i, j=1}^{2} \int_{\Omega} \frac{\partial u_{j}}{\partial x_{i}} \frac{\partial v_{j}}{\partial x_{i}} d x .
$$

It follows that $V \subset H \equiv H^{\prime} \subset V^{\prime}$, where the injections are dense and compact. We will use $\|\cdot\|_{*}$ for the norm in $V^{\prime}$ and $\langle\cdot, \cdot\rangle$ for the duality $\left\langle V^{\prime}, V\right\rangle$. Denote by $a(u, v)=((u, v))$, and define the tri-linear form $b$ on $V \times V \times V$ by

$$
b(u, v, w)=\sum_{i, j=1}^{2} \int_{\Omega} u_{i} \frac{\partial v_{j}}{\partial x_{i}} w_{j} d x \quad \forall u, v, w \in V .
$$

Some useful properties of this tri-linear form can be found in [4, p. 3182]. Here we only emphasize the ones which will be used in our stability analysis:

(I1) there exists $c_{1}:=c_{1}(\Omega)>0$ such that

$$
|b(u, v, w)| \leq c_{1}|u|^{1 / 2}\|u\|^{1 / 2}\|v\| \cdot|w|^{1 / 2}\|w\|^{1 / 2}, \quad \forall u, v, w \in V .
$$

(I2) there exists $c_{2}:=c_{2}(\Omega)>0$ such that

$$
|b(u, v, w)| \leq c_{2}|u|_{\left(L^{4}(\Omega)\right)^{2}}\|v\| \cdot\|w\|, \quad \forall u, v, w \in V .
$$

Let $X$ and $Y$ be two separable Banach spaces. In the sequel we will use the notation

$$
C_{X}:=C^{0}([-h, 0] ; X) ; \quad L_{X}^{2}:=L^{2}(-h, 0 ; X) .
$$

Given a function $u:[-h, T)] \rightarrow X$, for each $t \in(0, T)$ we denote by $u_{t}$ the function defined on $[-h, 0]$ via the relation

$$
u_{t}(s)=u(t+s), \quad s \in[-h, 0] .
$$

Before stating the problem in a suitable framework, we enumerate the standing assumptions on the term in which the delay is present. In general, consider $g$ : $[0, T] \times C_{X} \rightarrow Y$ such that

(I) for all $\xi \in C_{X}$, the mapping $t \in[0, T] \rightarrow g(t, \xi) \in Y$ is measurable,

(II) for each $t \in[0, T], g(t, 0)=0$,

(III) there exists $L_{g}>0$ such that $\forall t \in[0, T], \quad \forall \xi, \eta \in C_{X}$

$$
\|g(t, \xi)-g(t, \eta)\|_{Y} \leq L_{g}\|\xi-\eta\|_{C_{X}},
$$


(IV) there exists $C_{g}>0$ such that $\forall t \in[0, T], \forall u, v \in C^{0}([-h, T] ; X)$

$$
\int_{0}^{t}\left\|g\left(s, u_{s}\right)-g\left(s, v_{s}\right)\right\|_{Y}^{2} d s \leq C_{g} \int_{-h}^{t}\|u(s)-v(s)\|_{X}^{2} d s .
$$

Define the function $g_{u}: t \in[0, T] \rightarrow Y$ defined by $g_{u}(t)=g\left(t, u_{t}\right) \forall t \in[0, T]$. Observe that (I)-(III) imply the measurability of $g_{u}$ given $u \in C^{0}([-h, T] ; X)$ (see Bensoussan et al. [1] ) and $g_{u} \in L^{\infty}(0, T ; Y)$. Then thanks to (IV), the mapping

$$
\mathcal{G}: u \in C^{0}([-h, T] ; X) \rightarrow g_{u} \in L^{2}(0, T ; Y)
$$

possesses a unique extension to a mapping $\widetilde{\mathcal{G}}$ which is uniformly continuous from $L^{2}(-h, T ; X)$ into $L^{2}(0, T ; Y)$.

From now on, we will denote $g\left(t, u_{t}\right)=\widetilde{\mathcal{G}}(u)(t)$ for each $u \in L^{2}(-h, T ; X)$, and thus for any $t \in[0, T]$ and any $u, v \in L^{2}(-h, T ; X)$, we will have

$$
\int_{0}^{t}\left\|g\left(s, u_{s}\right)-g\left(s, v_{s}\right)\right\|_{Y}^{2} d s \leq C_{g} \int_{-h}^{t}\|u(s)-v(s)\|_{X}^{2} d s .
$$

With the convention above, assume that $u_{0} \in H, \phi \in L_{V}^{2}, f \in L^{2}\left(0, T ; V^{\prime}\right)$, $g_{1}:[0, T] \times C_{V} \rightarrow\left(L^{2}(\Omega)\right)^{2}$ satisfies hypotheses (I)-(IV) with $X=V, Y=\left(L^{2}(\Omega)\right)^{2}$, $L_{g_{1}}=L_{1}$ and $C_{g_{1}}=C_{1}$, and $g_{2}:[0, T] \times C_{V} \rightarrow V^{\prime}$ satisfies hypotheses (I)-(IV) with $X=V, Y=V^{\prime}, L_{g_{2}}=L_{2}$ and $C_{g_{2}}=C_{2}$.

We are interested in the following problem:

$$
\left\{\begin{array}{l}
\text { To find } u \in L^{2}(-h, T ; V) \cap L^{\infty}(0, T ; H) \text { such that, for all } v \in V, \\
\begin{array}{rl}
\frac{d}{d t}(u(t), v)+\nu a(u(t), v)+b(u(t), u(t), v)=\langle f(t), v\rangle+\left(g_{1}\left(t, u_{t}\right), v\right) \\
\quad+\left\langle g_{2}\left(t, u_{t}\right), v\right\rangle,
\end{array} \\
\begin{array}{rl}
u(0)=u_{0}, u(t)=\phi(t), \quad t \in(-h, 0), &
\end{array}
\end{array}\right.
$$

where the equation in (1) is understood in the distributional sense of $\mathcal{D}^{\prime}(0, T)$.

REMARK 1. Notice that all the terms in (1) are well defined. Particularly, thanks to assumptions (I)-(IV), if $u \in L^{2}(-h, T ; V)$ then the term $g_{1}\left(t, u_{t}\right)$ defines a function in $L^{2}\left(0, T ;\left(L^{2}(\Omega)^{2}\right)\right.$, and the term $g_{2}\left(t, u_{t}\right)$ defines a function in $L^{2}\left(0, T ; V^{\prime}\right)$. Thus if $u \in L^{2}(-h, T ; V) \cap L^{\infty}(0, T ; H)$ satisfies the equation in (1), $u$ is weakly continuous from $[0, T]$ into $H$ (see Lions $[\mathbf{1 1}]$ ), and therefore the initial condition $u(0)=u_{0}$ makes sense. If there exists a solution $u$ to the problem (1), then it belongs to the space $C^{0}([0, T] ; H)$.

In the next section we will review some results on the existence, uniqueness and regularity of solutions to (1).

\section{Existence, uniqueness and regularity of solutions}

In order to analyze the asymptotic behavior of our model in the 2-dimensional case, we first need to state a result on the existence and uniqueness of solutions. The first result below needs only assumptions (I)-(III), which allows for more general forms of the delay term. For instance, in the case of variable delay, only measurability of the delay is needed, while, in the general case which we will also include later on, assumption (IV) requires more regularity (e.g., continuous differentiability and boundedness on the derivative of the delay) but on the other side, the initial 
values must be continuous and not only square integrable (see García-Luengo et al. [8] for more details).

Let us first consider the following version of our model:

$$
\left\{\begin{array}{l}
\frac{\partial u}{\partial t}-\nu \Delta u+\sum_{i=1}^{2} u_{i} \frac{\partial u}{\partial x_{i}}=f-\nabla p+g\left(t, u_{t}\right) \quad \text { in }(0,+\infty) \times \Omega \\
\operatorname{div} u=0 \text { in }(0,+\infty) \times \Omega \\
u=0 \text { on }(0,+\infty) \times \Gamma \\
u(0, x)=u_{0}(x), \quad x \in \Omega, \\
u(t, x)=\phi(t, x), t \in(-h, 0), \quad x \in \Omega
\end{array}\right.
$$

Theorem 2. ([8]) Consider $\phi \in C_{H}$ with $\phi(0)=u_{0}, f \in L_{l o c}^{2}\left(\mathbb{R} ; V^{\prime}\right)$, and assume that $g: \mathbb{R} \times C_{H} \rightarrow\left(L^{2}(\Omega)\right)^{2}$ satisfies hypotheses (I)-(III) with $X=H$ and $Y=$ $\left(L^{2}(\Omega)\right)^{N}$. Then there exists a unique solution $u=u(\cdot ; \phi)$ of $(2)$, in other words, $u \in C^{0}([-h,+\infty) ; H)$ and $u \in L^{2}(0,+\infty ; V)$. Moreover, if $f \in L_{l o c}^{2}\left(\mathbb{R} ;\left(L^{2}(\Omega)\right)^{2}\right)$, then

(1) $u \in C^{0}([\varepsilon, T] ; V) \cap L^{2}(\varepsilon, T ; D(A))$ for all $T>\varepsilon>0$.

(2) If $\phi(0) \in V$, in fact $u$ is a strong solution of $(2)$, i.e. $u \in L^{2}(0, T ; D(A)) \cap$ $L^{\infty}(0, T ; V)$ for all $T>0$.

Proof. See García-Luengo et al. [8].

A more general result on the existence and uniqueness of solutions to the $2 \mathrm{D}$ model (1) was proved in [3] and is restated below in the two dimensional case.

Theorem 3. ([3]) Consider $u_{0} \in H, \phi \in L^{2}(-h, 0 ; V), f \in L^{2}\left(0, T ; V^{\prime}\right)$, and assume that $g_{1}:[0, T] \times C_{V} \rightarrow\left(L^{2}(\Omega)\right)^{2}$ satisfies hypotheses $(I)-(I V)$ with $X=V$, $Y=\left(L^{2}(\Omega)\right)^{2}, L_{g_{1}}=L_{1}$ and $C_{g_{1}}=C_{1}$, and $g_{2}:[0, T] \times C_{V} \rightarrow V^{\prime}$ satisfies hypotheses (I)-(IV) with $X=V, Y=V^{\prime}, L_{g_{2}}=L_{2}$ and $C_{g_{2}}=C_{2}$. Assume also that the following condition holds:

(C) If $v^{(m)}$ converges weakly to $v$ in $L^{2}(-h, T ; V)$ and strongly in $L^{2}(-h, T ; H)$, then $g_{i}\left(\cdot, v^{(m)}\right)$ converges weakly to $g_{i}(\cdot, v$. $)$ in $L^{2}\left(0, T ; V^{\prime}\right)$ for $i=1,2$.

Then there exists a unique solution to problem (1) provided $\nu^{2}>C_{2}$.

Proof. See Caraballo and Real $[\mathbf{3}]$.

We next exhibit a few examples of delay forcing terms which fall within the framework of our general set-up.

3.1. Forcing term with bounded variable delays. For a fixed $d \in \mathbb{N}$, let $G:[0, T] \times\left(\mathbb{R}^{2}\right)^{d} \rightarrow \mathbb{R}^{2}$ be a measurable function satisfying $G(t, 0)=0$ for all $t \in[0, T]$, and assume that there exists $L_{1}>0$ such that

$$
\left|G\left(t, u_{1}, \ldots, u_{d}\right)-G\left(t, v_{1}, \ldots, v_{d}\right)\right|_{\mathbb{R}^{2}} \leq L_{1}\left|\left(u_{1}, \ldots, u_{d}\right)-\left(v_{1}, \ldots, v_{d}\right)\right|_{\left(\mathbb{R}^{2}\right)^{d}}
$$

for all $\left(u_{1}, \ldots, u_{d}\right),\left(v_{1}, \ldots, v_{d}\right) \in\left(\mathbb{R}^{2}\right)^{d}$.

Consider $d$ number of functions $\rho_{i}(\cdot):[0,+\infty) \rightarrow[0, h](i=1, \ldots, d)$, with $h>0$, which will play the role of delay functions. First, we assume that $\rho_{i}(\cdot)$ is measurable for each of $i=1, \ldots, d$. Then, define

$$
g_{1}\left(t, \xi_{1}, \ldots, \xi_{d}\right)(x)=G\left(t, \xi_{1}\left(-\rho_{1}(t)\right)(x), \ldots, \xi_{d}\left(-\rho_{d}(t)\right)(x)\right)
$$


for $\xi_{i} \in C^{0}([0, T] ; H), x \in \Omega, t \in[0, T]$. Notice that in this case, the delayed term $g_{1}$ in our problem becomes

$$
g_{1}\left(t, u_{t}\right)=G\left(t, u\left(t-\rho_{1}(t)\right), \ldots, u\left(t-\rho_{d}(t)\right)\right) .
$$

Then, $g_{1}$ satisfies the hypotheses in Theorem 2 with $X=H$ and $Y=L^{2}(\Omega)^{2}$, since (I)-(III) follow immediately.

However, in order to apply Theorem 3 we need to impose stronger assumptions on the delay functions. Indeed, for example when $d=1$ we assume that $\rho \in C^{1}([0, T]), \rho(t) \geq 0$ for all $t \in[0, T], h=\max _{t \in[0, T]} \rho(t)>0$ and $\rho_{*}=$ $\max _{t \in[0, T]} \rho^{\prime}(t)<1$. For $u, v \in L^{2}(-h, T ; H)$, using the change of variable $\tau=$ $s-\rho(s)$ gives directly that

$$
\int_{0}^{t}\left|g_{1}\left(s, u_{s}\right)-g_{1}\left(s, v_{s}\right)\right|^{2} d s \leq \frac{L_{1}^{2}}{1-\rho_{*}} \int_{-h}^{t}|u(\tau)-v(\tau)|^{2} d \tau \quad \forall t \in[0, T],
$$

and, consequently (IV) and (C) are fulfilled.

3.2. Forcing term with distributed delay. Let $G:[0, T] \times[-h, 0] \times \mathbb{R}^{2} \rightarrow$ $\mathbb{R}^{2}$ be a measurable function satisfying $G(t, s, 0)=0$ for all $(t, s) \in[0, T] \times[-h, 0]$ and such that there exists a function $\gamma \in L^{2}(-h, 0)$ with

$$
|G(t, s, u)-G(t, s, v)|_{\mathbb{R}^{2}} \leq \gamma(s)|u-v|_{\mathbb{R}^{2}}, \forall u, v \in \mathbb{R}^{2}, \quad \forall(t, s) \in[0, T] \times[-h, 0] .
$$

Then, defining $g_{1}(t, \xi)(x)=\int_{-h}^{0} G(t, s, \xi(s)(x)) d s$ for each $\xi \in C^{0}([0, T] ; H), x \in \Omega$ and $t \in[0, T]$, the delayed term $g_{1}$ becomes

$$
g_{1}\left(t, u_{t}\right)=\int_{-h}^{0} G(t, s, u(t+s)) d s .
$$

As in subsection 3.1, $g_{1}$ satisfies the hypotheses in Theorem 2 with $X=H$ and $Y=\left(L^{2}(\Omega)\right)^{2}$ (see Caraballo and Real $[\mathbf{3}]$ ).

\section{Existence and exponential stability of stationary solutions}

In this section we will analyze the long time behavior of solutions in a neighborhood of a stationary solution to the model in two dimensional case. First we will recall a general result ensuring the existence and uniqueness of such stationary solution. Then we will prove several stability results for the stationary solutions which will improve some existing ones in the literature.

Our first result generalizes Theorem 3.3 in [4] and Theorem 3.1 in [13] (where the stationary solution is the zero solution), in the sense that our new result is valid for general delay terms rather than only variable delays as in $[\mathbf{4}]$ and $[\mathbf{1 3}]$. In our second result we will consider a case in which $f \in V^{\prime}$. Our last main result provides an improvement to the deterministic counterpart of the stability analysis on a stochastic Navier-Stokes model considered in [6]. More precisely, Chen shows in [6] a sufficient condition for the exponential stability of the stationary solution of a stochastic two-dimensional Navier-Stokes perturbed by variable delay terms, which also provides a sufficient condition for the deterministic case. Our goal is to prove a more general result which is valid for more general types of delay terms. 
4.1. Existence and uniqueness of stationary solutions. Our next analysis will be carried out in an abstract formulation for our problem. To this end, we define the operator $A: V \rightarrow V^{\prime}$ by

$$
\langle A u, v\rangle=((u, v)), \quad \forall u, v \in V,
$$

consider the operator $B: V \times V \rightarrow V^{\prime}$ defined by

$$
\langle B(u, v), w\rangle=b(u, v, w), \quad \forall u, v, w \in V,
$$

and set $B(u)=B(u, u)$. Then, (2) can be rewritten as

$$
\left\{\begin{array}{l}
\frac{\mathrm{d}}{\mathrm{d} t} u(t)+\nu A u(t)+B(u(t))=f(t)+g\left(t, u_{t}\right) \text { in } V^{\prime}, \\
u(0)=u_{0}, \quad u(t)=\phi(t), \quad t \in(-h, 0),
\end{array}\right.
$$

Observe that if we denote $D(A)=\left(H^{2}(\Omega)\right)^{2} \cap V$, then

$$
A u=-P \Delta u, \quad \forall u \in D(A),
$$

where $P$ is the orthogonal projector from $\left(L^{2}(\Omega)\right)^{2}$ onto $H$ and, in the sequel, $\lambda_{1}$ will denote the first eigenvalue of $A$.

Then, we will next consider the following equation

$$
\frac{d u}{d t}+\nu A u+B(u)=f+g\left(t, u_{t}\right)
$$

with $f \in V^{\prime}$ independent of $t$. A stationary solution to (4) is $u^{*} \in V$ such that

$$
\nu A u^{*}+B\left(u^{*}\right)=f+g\left(t, \hat{u}^{*}\right)
$$

for all $t \geq 0$, where for any $u^{*} \in H$, the function $\hat{u}^{*}(\cdot):[-h, 0] \rightarrow H$ denotes the constant function defined by $\hat{u}^{*}(\theta)=u^{*}$ for all $\theta \in[-h, 0]$.

In the papers $[4]$ (for the deterministic case) and [6] (for a stochastic version) this problem is analyzed by assuming that the delay term has the special form

$$
g\left(t, u_{t}\right)=G(u(t-\rho(t))),
$$

where $G: \mathbb{R}^{2} \rightarrow \mathbb{R}^{2}$ is a function satisfying $G(0)=0$ and such that there exists $L_{1}>0$ for which

$$
|G(u)-G(v)|_{\mathbb{R}^{2}} \leq L_{1}|u-v|_{\mathbb{R}^{2}}, \quad \forall u, v \in \mathbb{R}^{2} .
$$

In [4] the term $\rho$ satisfies $\rho \in C^{1}([0,+\infty))$ and

$$
\rho(t) \geq 0, \forall t \geq 0, h=\sup _{t \geq 0} \rho(t) \in(0,+\infty), \rho_{*}=\sup _{t \geq 0} \rho^{\prime}(t)<1,
$$

while in [6] only measurability on $\rho$ is needed. In Taniguchi $[\mathbf{1 3}]$, the function $G$ is allowed to be dependent on the time variable, i.e., it has the form $G(t, u(t-\rho(t)))$, being only locally Lipschitz but satisfying a sub-linear growth condition, and the delay $\rho \in C^{1}([0,+\infty))$ satisfying the assumption (5) as well.

Next we will improve the stability results proved in $[4, \mathbf{1 3}]$ in the sense that it will hold not only for variable delays, but also for an abstract functional setup which covers a wider variety of delays (for instance, the delays considered in subsections 3.1 and 3.2 can be included in this general set-up by assuming that the function $G(\cdot)$ is independent of the variable $t$ ). 
In fact, to carry out our analysis, we assume that there exists a function $G$ : $H \rightarrow H$ such that

$$
g\left(t, \hat{\xi}^{*}\right)=G\left(\xi^{*}\right), \text { for all } t \in \mathbb{R}, \text { and all } \xi^{*} \in H,
$$

and there exists $L_{1}>0$ such that

$$
|G(u)-G(v)|_{H} \leq L_{1}|u-v|_{H}, \quad \forall u, v \in H .
$$

In the next theorem we will establish a result on the existence and uniqueness of stationary solutions to our equation (4), i.e., there exists $u^{*} \in V$ such that

$$
\nu A u^{*}+B\left(u^{*}\right)=f+g\left(t, \hat{u}^{*}\right) \text { for all } t \geq 0,
$$

or, in other words,

$$
\nu A u^{*}+B\left(u^{*}\right)=f+G\left(u^{*}\right) .
$$

THEOREM 4. Assume that $G$ satisfies the conditions (6) - (7) as stated above and $\nu>\lambda_{1}^{-1} L_{1}$. Then,

(a) for all $f \in V^{\prime}$ there exists a stationary solution to (4);

(b) if $f \in\left(L^{2}(\Omega)\right)^{2}$, the stationary solutions belong to $D(A)$, the domain of operator $A$;

(c) there exists a constant $c_{3}(\Omega)>0$ and if $\nu$ satisfies the inequality $(\nu-$ $\left.\lambda_{1}^{-1} L_{1}\right)^{2}>c_{3}(\Omega)\|f\|_{V^{\prime}}$, then the stationary solution to (4) is unique, where $c_{3}(\Omega)>0$ is determined in the proof.

Proof. Although the proof follows the same lines as in Caraballo and Real [4] for the case with variable delay, we prefer to include it here for the sake of completeness.

(a) By the Lax-Milgram Theorem, for each $z \in V$, there exists a unique $u \in V$ such that

$$
\nu a(u, v)+b(z, u, v)=\langle f, v\rangle+(G(z), v), \quad \forall v \in V .
$$

Taking $v=u$ in (8), it follows that

$$
\nu\|u\| \leq\|f\|_{V^{\prime}}+\lambda_{1}^{-1} L_{1}\|z\| .
$$

Let us pick $k>0$ such that $k\left(\nu-\lambda_{1}^{-1} L_{1}\right) \geq\|f\|_{V^{\prime}}$, and denote

$$
\mathcal{C}=\{z \in V ;\|z\| \leq k\} .
$$

Then, $\mathcal{C}$ is a convex and compact subset of $\left(L^{4}(\Omega)\right)^{2}$, and by $(9)$, the mapping $z \mapsto u$, defined by (8), maps $\mathcal{C}$ into $\mathcal{C}$. If we prove that this mapping is continuous in $\mathcal{C}$ with the topology induced by $\left(L^{4}(\Omega)\right)^{2}$, then the Schauder Theorem implies the existence of a fixed point in $\mathcal{C}$, and obviously this fixed point is a stationary solution to (4). In fact, the continuity of $z \mapsto u$ can be shown as follows. Let $z_{i} \in \mathcal{C}$ and $u_{i} \in \mathcal{C}$ be such that

$$
\nu a\left(u_{i}, v\right)+b\left(z_{i}, u_{i}, v\right)=\langle f, v\rangle+\left(G\left(z_{i}\right), v\right), \quad \forall v \in V, \quad i=1,2 .
$$

Then, by (I2) and (7),

$$
\begin{aligned}
\nu\left\|u_{1}-u_{2}\right\|^{2}= & b\left(z_{1}-z_{2}, u_{1}, u_{1}-u_{2}\right)+\left(G\left(z_{1}\right)-G\left(z_{2}\right), u_{1}-u_{2}\right) \\
\leq & k c_{2}(\Omega)\left|z_{1}-z_{2}\right|_{\left.L^{4}(\Omega)\right)^{2}}\left\|u_{1}-u_{2}\right\| \\
& \quad+L_{1} \lambda_{1}^{-1 / 2}\left|z_{1}-z_{2}\right|\left\|u_{1}-u_{2}\right\| .
\end{aligned}
$$

As $V \subset\left(L^{4}(\Omega)\right)^{2}$ and $\left(L^{4}(\Omega)\right)^{2} \subset\left(L^{2}(\Omega)\right)^{2}$ with continuous injections, the continuity of the mapping $z \mapsto u$ in $\mathcal{C}$ follows from (10). 
(b) If $f \in\left(L^{2}(\Omega)\right)^{2}$, then every stationary solution $u^{*}$ to (4) is also a solution to (1), but with initial data $u_{0}=\phi(t)=u^{*}$ for $t \in[-h, 0)$, and forcing term $\tilde{f}=P\left(f+G\left(u^{*}\right)\right) \in H \subset L^{2}(0, T ; H)$. Thus, the standard regularity results from the theory of the Navier-Stokes equations without delays can be applied (see [4]).

(c) Let $f \in V^{\prime}$ and $u_{1}$ and $u_{2}$ be the stationary solutions to (4). Then by the same argument as for inequality (10),

$$
\begin{gathered}
\nu\left\|u_{1}-u_{2}\right\|^{2} \leq \quad c_{2}(\Omega)\left|u_{1}-u_{2}\right|_{\left.L^{4}(\Omega)\right)^{2}}\left\|u_{1}\right\|\left\|u_{1}-u_{2}\right\| \\
+L_{1} \lambda_{1}^{-1 / 2}\left|u_{1}-u_{2}\right|\left\|u_{1}-u_{2}\right\| .
\end{gathered}
$$

Since

$$
\nu\left\|u_{1}\right\|^{2}=\left\langle f, u_{1}\right\rangle+\left(G\left(u_{1}\right), u_{1}\right) \leq\|f\|_{V^{\prime}}\left\|u_{1}\right\|+\lambda_{1}^{-1} L_{1}\left\|u_{1}\right\|^{2},
$$

then

$$
\left(\nu-\lambda_{1}^{-1} L_{1}\right)\left\|u_{1}\right\| \leq\|f\|_{V^{\prime}} .
$$

By the continuous injection of $V$ into $\left(L^{4}(\Omega)\right)^{2}$, there exists a constant $c_{*}>0$ such that $\left|u_{1}-u_{2}\right|_{\left(L^{4}(\Omega)\right)^{2}} \leq c_{*}\left\|u_{1}-u_{2}\right\|$. Set $c_{3}(\Omega)=c_{*} c_{2}(\Omega)$. Then we obtain from (11) that

$$
\left(\nu-\lambda_{1}^{-1} L_{1}\right)^{2}\left\|u_{1}-u_{2}\right\|^{2} \leq c_{3}(\Omega)\|f\|_{V^{\prime}}\left\|u_{1}-u_{2}\right\|^{2} .
$$

This completes the proof.

4.2. Exponential convergence of solutions: a first general result by a direct approach. Let us now prove that, under appropriate assumptions, our model possesses a unique stationary solution, $u_{\infty}$, and every weak solution approaches $u_{\infty}$ exponentially fast as $t$ goes to $+\infty$. First we consider the case in which $f \in\left(L^{2}(\Omega)\right)^{2}$ yielding the existence of stationary solutions in $D(A)$.

THEOREM 5. Assume that the forcing term $g(\cdot, \cdot)$ satisfies conditions (I)-(III) with $X=H$ and $Y=\left(L^{2}(\Omega)\right)^{2}$, and for every $\varepsilon>0$ small enough, and any $u, v \in C([-h,+\infty) ; H)$, it holds

$$
\int_{0}^{t} e^{\varepsilon s}\left|g\left(s, u_{s}\right)-g\left(s, v_{s}\right)\right|^{2} d s \leq C_{g}^{2} \int_{-h}^{t} e^{\varepsilon s}|u(s)-v(s)|^{2} d s,
$$

where $C_{g}$ only depends on $h$ and $\varepsilon$.

Assume in addition that there exist two constants $k_{i}>0, i=1,2$, depending only on $\Omega$, such that if $f \in\left(L^{2}(\Omega)\right)^{2}$ and $\nu>\lambda_{1}^{-1} L_{1}$ it holds

$$
2 \nu \lambda_{1}>2 C_{g}+\frac{k_{1}|f|}{\nu-\lambda_{1}^{-1} L_{1}}+\frac{k_{2}|f|^{3}}{\nu^{2}\left(\nu-\lambda_{1}^{-1} L_{1}\right)^{3}} .
$$

Then there is a unique stationary solution $u_{\infty} \in D(A)$ of (4) and every solution of (1) converges to $u_{\infty}$ exponentially fast as $t \rightarrow+\infty$. More precisely, there exist two positive constants $C$ and $\lambda$, such that for all $u_{0} \in H$ and $\phi \in L_{V}^{2}$, the solution $u$ of (1) with $f(t) \equiv f$ satisfies

$$
\left|u(t)-u_{\infty}\right|^{2} \leq C e^{-\lambda t}\left(\left|u_{0}-u_{\infty}\right|^{2}+\left\|\phi-\hat{u}_{\infty}\right\|_{L_{V}^{2}}^{2}\right), \quad \forall t \geq 0 .
$$


Proof. Assume that $f \in\left(L^{2}(\Omega)\right)^{2}$, and consider $u(\cdot)$, the solution of $(2)$ for $f(t) \equiv f$, and let $u_{\infty} \in D(A)$ be a stationary solution to (4) whose existence is ensured by Theorem 4 . Let us write $w(t)=u(t)-u_{\infty}$, and observe that

$$
\frac{\mathrm{d}}{\mathrm{d} t} w(t)+\nu A w(t)+B(u(t))-B\left(u_{\infty}\right)=g\left(t, u_{t}\right)-g\left(t, \hat{u}_{\infty}\right) .
$$

Thanks to (14), we can choose $\lambda>0$, small enough, such that

$$
\lambda+2 C_{g}-2 \nu \lambda_{1}+\frac{k_{1}|f|}{\left(\nu-L_{1} \lambda_{1}^{-1}\right)}+\frac{k_{2}|f|^{3}}{\nu^{2}\left(\nu-L_{1} \lambda_{1}^{-1}\right)^{3}} \leq 0 .
$$

Now, for such a $\lambda$, by standard computations we obtain

$$
\begin{aligned}
\frac{\mathrm{d}}{\mathrm{d} t}\left(\mathrm{e}^{\lambda t}|w(t)|^{2}\right)= & \lambda \mathrm{e}^{\lambda t}|w(t)|^{2}+\mathrm{e}^{\lambda t} \frac{\mathrm{d}}{\mathrm{d} t}|w(t)|^{2} \\
\leq & \mathrm{e}^{\lambda t}\left[\lambda|w(t)|^{2}-2 \nu\|w(t)\|^{2}+2 b\left(w(t), w(t), u_{\infty}\right)\right. \\
& \left.+2\left|g\left(t, u_{t}\right)-g\left(t, \hat{u}_{\infty}\right)\right| \cdot|w(t)|\right] \\
\leq & \lambda_{1}^{-1} \mathrm{e}^{\lambda t}\left(\lambda+C_{g}-2 \nu \lambda_{1}\right)\|w(t)\|^{2} \\
& +2 \mathrm{e}^{\lambda t}\left|b\left(w(t), w(t), u_{\infty}\right)\right|+\frac{1}{C_{g}} \mathrm{e}^{\lambda t}\left|g\left(t, u_{t}\right)-g\left(t, \hat{u}_{\infty}\right)\right|^{2} .
\end{aligned}
$$

Obviously

$$
\left|b\left(w(t), w(t), u_{\infty}\right)\right| \leq c|w(t)| \cdot\|w(t)\| \cdot\left|u_{\infty}\right|_{\infty},
$$

where we denote by $\left|u_{\infty}\right|_{\infty}$ the norm of $u_{\infty}$ in $\left(L^{\infty}(\Omega)\right)^{2}$. Observe that $H^{2}(\Omega) \subset$ $L^{\infty}(\Omega)$ with continuous injection, and that there exists a constant $C(\Omega)>0$ such that

$$
|u|_{\left(H^{2}(\Omega)\right)^{2}} \leq C(\Omega)|A u|, \forall u \in D(A)=\left(H^{2}(\Omega)\right)^{2} \cap V .
$$

Thus we obtain the existence of a constant $d_{1}>0$ depending only on $\Omega$ such that

$$
\left|b\left(w(t), w(t), u_{\infty}\right)\right| \leq d_{1} \lambda_{1}^{-1 / 2}\|w(t)\|^{2}\left|A u_{\infty}\right| .
$$

On the other hand,

$$
\begin{aligned}
\nu\left|A u_{\infty}\right| & \leq|f|+\left|g\left(t, u_{\infty}\right)\right|+\left|B\left(u_{\infty}\right)\right| \\
& \leq|f|+\left|G\left(u_{\infty}\right)\right|+\left|B\left(u_{\infty}\right)\right| \\
& \leq|f|+L_{1}\left|u_{\infty}\right|+c^{\prime}\left\|u_{\infty}\right\| \cdot\left|u_{\infty}\right|_{\infty},
\end{aligned}
$$

and consequently, from the continuous injection of $H^{2}(\Omega)$ into $L^{\infty}(\Omega)$, the inequality (19), and the Gagliardo-Nirenberg interpolation inequality, we obtain

$$
\nu\left|A u_{\infty}\right| \leq|f|+L_{1}\left|u_{\infty}\right|+c^{\prime \prime}|| u_{\infty} \|\left|u_{\infty}\right|^{1 / 2}\left|A u_{\infty}\right|^{1 / 2}
$$

Noticing that

$$
c^{\prime \prime}\left\|u_{\infty}\right\|\left|u_{\infty}\right|^{1 / 2}\left|A u_{\infty}\right|^{1 / 2} \leq \frac{\left(c^{\prime \prime}\right)^{2} \lambda_{1}^{-1 / 2}}{2 \nu}\left\|u_{\infty}\right\|^{3}+\frac{\nu}{2}\left|A u_{\infty}\right|
$$

from (21) we deduce

$$
\left|A u_{\infty}\right| \leq \frac{2}{\nu}|f|+\frac{2 L_{1} \lambda_{1}^{-1 / 2}}{\nu}\left\|u_{\infty}\right\|+\frac{\left(c^{\prime \prime}\right)^{2} \lambda_{1}^{-1 / 2}}{\nu^{2}}\left\|u_{\infty}\right\|^{3} .
$$

Now, as

$$
\begin{aligned}
& \nu\left\|u_{\infty}\right\|^{2}=\left(f, u_{\infty}\right)+\left(G\left(u_{\infty}\right), u_{\infty}\right) \\
& \leq|f| \lambda_{1}^{-1 / 2}\left\|u_{\infty}\right\|+L_{1} \lambda_{1}^{-1}\left\|u_{\infty}\right\|^{2},
\end{aligned}
$$


we obtain from (22) that

$$
\begin{aligned}
\left|A u_{\infty}\right| & \leq \frac{2}{\nu}|f|+\frac{2 L_{1} \lambda_{1}^{-1}}{\nu\left(\nu-L_{1} \lambda_{1}^{-1}\right)}|f|+\frac{\left(c^{\prime \prime}\right)^{2} \lambda_{1}^{-2}}{\nu^{2}\left(\nu-L_{1} \lambda_{1}^{-1}\right)^{3}}|f|^{3} \\
& =\frac{2}{\left(\nu-L_{1} \lambda_{1}^{-1}\right)}|f|+\frac{\left(c^{\prime \prime}\right)^{2} \lambda_{1}^{-2}}{\nu^{2}\left(\nu-L_{1} \lambda_{1}^{-1}\right)^{3}}|f|^{3} .
\end{aligned}
$$

From (17), (20), (23), and denoting

$$
k_{1}=4 d_{1} \lambda_{1}^{1 / 2}, \quad k_{2}=2 d_{1} \lambda_{1}^{-3 / 2}\left(c^{\prime \prime}\right)^{2},
$$

it follows that

$$
\begin{aligned}
& \frac{\mathrm{d}}{\mathrm{d} t}\left(\mathrm{e}^{\lambda t}|w(t)|^{2}\right) \\
& \leq \lambda_{1}^{-1} \mathrm{e}^{\lambda t}\left(\lambda+C_{g}-2 \nu \lambda_{1}+\frac{k_{1}|f|}{\left(\nu-L_{1} \lambda_{1}^{-1}\right)}+\frac{k_{2}|f|^{3}}{\nu^{2}\left(\nu-L_{1} \lambda_{1}^{-1}\right)^{3}}\right)\|w(t)\|^{2} \\
& \quad+\frac{1}{C_{g}} \mathrm{e}^{\lambda t}\left|g\left(t, u_{t}\right)-g\left(t, \hat{u}_{\infty}\right)\right|^{2} .
\end{aligned}
$$

Integrating (24) over the interval $[0, t]$, and taking into account (13) and (16), we deduce

$$
\mathrm{e}^{\lambda t}|w(t)|^{2} \leq|w(0)|^{2}+C_{g} \int_{-h}^{0} \mathrm{e}^{\lambda s}|w(s)|^{2} \mathrm{~d} s,
$$

and hence (15) is satisfied. The uniqueness of $u_{\infty}$ follows from the fact that if $v_{\infty}$ is another stationary solution of (4), then the function defined as $u(t) \equiv v_{\infty}$, for all $t \geq-h$, is a solution of (1) with $u_{0}=v_{\infty}$, and $\phi=v_{\infty}$, and consequently, applying (15) and letting $t \rightarrow+\infty$, one has $\left|v_{\infty}-u_{\infty}\right|^{2} \leq 0$.

REMARK 6. In the particular case of variable delay considered in [4] in which $g\left(t, u_{t}\right)=G\left(u(t-\rho(t))\right.$, with $G$ globally Lipschitz with constant $L_{1}$, and $\rho \in$ $C^{1}([0,+\infty)), \quad \rho(t) \geq 0$ for all $t \geq 0, h=\sup _{t \geq 0} \rho(t) \in(0,+\infty)$ and $\rho_{*}=$ $\sup _{t \geq 0} \rho^{\prime}(t)<1$. Then, we obtain

$$
\begin{aligned}
\int_{0}^{t} e^{\lambda s}\left|g\left(s, u_{s}\right)-g\left(s, v_{s}\right)\right|^{2} d s & \left.\leq L_{1}^{2} \int_{0}^{t} e^{\lambda s} \mid u(s-\rho(s))-v(s-\rho(s))\right)\left.\right|^{2} d s \\
& \leq \frac{e^{\lambda h} L_{1}^{2}}{1-\rho_{*}} \int_{-h}^{t} e^{\lambda s}|u(s)-v(s)|^{2} d s,
\end{aligned}
$$

and therefore

$$
C_{g}^{2}=\frac{e^{\lambda h} L_{1}^{2}}{1-\rho_{*}}
$$

In this way, assumption (14) would become

$$
2 \nu \lambda_{1}>\frac{2 e^{\lambda h / 2} L_{1}}{\left(1-\rho_{*}\right)^{1 / 2}}+\frac{k_{1}|f|}{\nu-\lambda_{1}^{-1} L_{1}}+\frac{k_{2}|f|^{3}}{\nu^{2}\left(\nu-\lambda_{1}^{-1} L_{1}\right)^{3}} .
$$

However, if we assume that

$$
2 \nu \lambda_{1}>\frac{2 L_{1}}{\left(1-\rho_{*}\right)^{1 / 2}}+\frac{k_{1}|f|}{\nu-\lambda_{1}^{-1} L_{1}}+\frac{k_{2}|f|^{3}}{\nu^{2}\left(\nu-\lambda_{1}^{-1} L_{1}\right)^{3}},
$$

then there exists $\lambda_{0}>0$ such that for every $0<\lambda<\lambda_{0}$, it holds (26), which implies the exponential convergence to the stationary solution $u_{\infty}$ of the solution $u(\cdot)$. 
Comparing (27) with the sufficient condition established in [4], which reads as

$$
2 \nu \lambda_{1}>\frac{\left(2-\rho_{*}\right) L_{1}}{1-\rho_{*}}+\frac{k_{1}|f|}{\nu-\lambda_{1}^{-1} L_{1}}+\frac{k_{2}|f|^{3}}{\nu^{2}\left(\nu-\lambda_{1}^{-1} L_{1}\right)^{3}},
$$

it is straightforward to check that condition (27) is weaker than (28). Indeed, it holds that

$$
\frac{2 L_{1}}{\left(1-\rho_{*}\right)^{1 / 2}}<\frac{\left(2-\rho_{*}\right) L_{1}}{1-\rho_{*}}, \text { for all } \rho_{*} \in(0,1)
$$

and this implies that (27) holds for a larger range of values of $\nu$. For instance, if $\rho_{*}=0.99$, then $\frac{2}{\left(1-\rho_{*}\right)^{1 / 2}}=20$ and $\frac{\left(2-\rho_{*}\right)}{1-\rho_{*}}=100$.

If we assume that $f=0$, then our situation is within the framework covered by Theorem 3.1 in $[\mathbf{1 3}]$, and it is straightforward to check that condition (27) is exactly the same as imposed in [13, Theorem 3.1].

If we assume in Theorem 5 that the term $f \in V^{\prime}$, a similar but simpler proof yields to the following result.

THEOREM 7. Assume that the forcing term $g(\cdot, \cdot)$ satisfies conditions (I)-(III) with $X=H$ and $Y=\left(L^{2}(\Omega)\right)^{2}$, and for every $\varepsilon>0$ small enough and any $u, v \in C([-h,+\infty) ; H)$, it holds

$$
\int_{0}^{t} e^{\varepsilon s}\left|g\left(s, u_{s}\right)-g\left(s, v_{s}\right)\right|^{2} d s \leq C_{g}^{2} \int_{-h}^{t} e^{\varepsilon s}|u(s)-v(s)|^{2} d s
$$

where $C_{g}$ only depends on $h$ and $\varepsilon$.

Also assume that $f \in V^{\prime}, \nu>\lambda_{1}^{-1} L_{1}$ and

$$
\nu \lambda_{1}>C_{g}+\frac{c_{1}\|f\|_{*}}{\nu-L_{1} \lambda_{1}^{-1}} .
$$

Then there is a unique stationary solution $u_{\infty} \in V$ of (4) and every solution of (1) converges to $u_{\infty}$ exponentially fast as $t \rightarrow+\infty$.

Proof. The proof is simpler in this case as we can use directly estimate (I1) to obtain

$$
\left|b\left(w(t), w(t), u_{\infty}\right)\right| \leq c_{1} \lambda_{1}^{-1 / 2}\|w(t)\|^{2}\left\|u_{\infty}\right\| .
$$

Then, on the other hand,

$$
\begin{aligned}
\nu\left\|u_{\infty}\right\|^{2} & =\left\langle f, u_{\infty}\right\rangle+\left(G\left(u_{\infty}\right), u_{\infty}\right) \\
& \leq\|f\|_{*}\left\|u_{\infty}\right\|+L_{1} \lambda_{1}^{-1}\left\|u_{\infty}\right\|^{2},
\end{aligned}
$$

and therefore

$$
\left\|u_{\infty}\right\| \leq \frac{\|f\|_{*}}{\nu-L_{1} \lambda_{1}^{-1}} .
$$

Taking into account these estimates in (17) and arguing as in the previous proof, the result follows immediately. 
4.3. Exponential stability via a Gronwall-like lemma. In this subsection we aim at improving a sufficient condition for the exponential stability of stationary solutions to the Navier-Stokes model with delay, which could be implied by the analysis carried out in [6] for its stochastic counterpart. However, in [6] only the case of variable delay was considered while here we will extend the results to a much more general framework. The main tool utilized here is a Gronwall-like lemma which was proved in $[\mathbf{6}]$ and is recalled below.

Lemma 8. ([6, Lemma 3.2]) Let $y(\cdot):[-h,+\infty) \rightarrow[0,+\infty)$ be a function. Assume that there exist positive numbers $\gamma, \alpha_{1}$ and $\alpha_{2}$ such that $\gamma>\alpha_{2}$, and the following inequality holds:

$$
y(t) \leq \begin{cases}\alpha_{1} e^{-\gamma t}+\alpha_{2} \int_{0}^{t} e^{-\gamma(t-s)} \sup _{\theta \in[-h, 0]} y(s+\theta) d s, & t \geq 0, \\ \alpha_{1} e^{-\gamma t}, & t \in[-h, 0]\end{cases}
$$

Then,

$$
y(t) \leq \alpha_{1} e^{-\mu t}, \text { for } t \geq-h,
$$

where $\mu \in(0, \gamma)$ is given by the unique root of the equation

$$
\frac{\alpha_{2}}{\gamma-\mu} e^{\mu h}=1
$$

in this interval.

We state our stability result in the next theorem.

THEOREM 9. Assume that $g(\cdot, \cdot)$ satisfies conditions (I)-(III) with $X=H$ and $Y=\left(L^{2}(\Omega)\right)^{2}$ and $f \in V^{\prime}$. Assume also that $u_{\infty} \in V$ is the unique stationary solution to (4). Then the unique stationary solution $u_{\infty}$ to the model (1) is exponentially stable, provided

$$
\nu>\frac{c_{1}}{\sqrt{\lambda_{1}}}\left\|u_{\infty}\right\|+\frac{L_{g}}{\lambda_{1}} .
$$

Proof. Let us first choose a positive constant $\lambda>0$ such that

$$
\lambda-\lambda_{1}\left(2 \nu-\frac{2 c_{1}}{\sqrt{\lambda_{1}}}\left\|u_{\infty}\right\|\right)>0 .
$$

Then, for a weak solution $u(\cdot)$ to the model (1) corresponding to the initial datum $\phi$, taking into account condition (III) and the fact that $\nu A u_{\infty}+B u_{\infty}-f-g\left(t, u_{\infty}\right)=0$, we obtain, for $t \geq 0$,

$$
\begin{aligned}
\frac{d}{d t}\left(e^{\lambda t}\left|u(t)-u_{\infty}\right|^{2}\right)= & \lambda e^{\lambda t}\left|u(t)-u_{\infty}\right|^{2}+e^{\lambda t} \frac{d}{d t}\left|u(t)-u_{\infty}\right|^{2} \\
= & \lambda e^{\lambda t}\left|u(t)-u_{\infty}\right|^{2}+2 e^{\lambda t}\left\langle\frac{d}{d t}\left(u(t)-u_{\infty}\right), u(t)-u_{\infty}\right\rangle \\
= & \lambda e^{\lambda t}\left|u(t)-u_{\infty}\right|^{2}+2 e^{\lambda t}\left\langle-\nu A\left(u(t)-u_{\infty}\right), u(t)-u_{\infty}\right\rangle \\
& +2 e^{\lambda t}\left\langle-\left(B u(t)-B u_{\infty}\right), u(t)-u_{\infty}\right\rangle \\
& +2 e^{\lambda t}\left(g\left(t, u_{t}\right)-g\left(t, u_{\infty}\right), u(t)-u_{\infty}\right) \\
\leq & \lambda e^{\lambda t}\left|u(t)-u_{\infty}\right|^{2}-2 e^{\lambda t} \nu\left\|u(t)-\hat{u}_{\infty}\right\|^{2} \\
& +2 e^{\lambda t}\left|b\left(u(t)-u_{\infty}, u_{\infty}, u(t)-u_{\infty}\right)\right|+2 e^{\lambda t} L_{g}\left\|u_{t}-u_{\infty}\right\|_{C_{H}}^{2}
\end{aligned}
$$


According (I1), it follows

$$
\left|b\left(u(t)-u_{\infty}, u_{\infty}, u(t)-u_{\infty}\right)\right| \leq \frac{c_{1}}{\sqrt{\lambda_{1}}}\left\|u_{\infty}\right\|\left\|u(t)-u_{\infty}\right\|^{2},
$$

and we arrive at

$$
\begin{aligned}
\frac{d}{d t}\left(e^{\lambda t}\left|u(t)-u_{\infty}\right|^{2}\right) \leq & \lambda e^{\lambda t}\left|u(t)-u_{\infty}\right|^{2}-2 e^{\lambda t} \nu\left\|u(t)-u_{\infty}\right\|^{2} \\
& +e^{\lambda t} \frac{2 c_{1}}{\sqrt{\lambda_{1}}}\left\|u_{\infty}\right\|\left\|u(t)-u_{\infty}\right\|^{2}+2 e^{\lambda t} L_{g}\left\|u_{t}-u_{\infty}\right\|_{C_{H}}^{2} \\
(34) \leq & e^{\lambda t}\left[\lambda+\lambda_{1}\left(-2 \nu+\frac{2 c_{1}}{\sqrt{\lambda_{1}}}\left\|u_{\infty}\right\|\right)\right]\left|u(t)-u_{\infty}\right|^{2} \\
& +2 e^{\lambda t} L_{g}\left\|u_{t}-u_{\infty}\right\|_{C_{H}}^{2} \\
\leq & e^{\lambda t}\left[\lambda+\lambda_{1}\left(-2 \nu+\frac{2 c_{1}}{\sqrt{\lambda_{1}}}\left\|u_{\infty}\right\|+\frac{2 L_{g}}{\lambda_{1}}\right]\right)\left\|u_{t}-u_{\infty}\right\|_{C_{H}}^{2} .
\end{aligned}
$$

Integrating $(35)$ over the interval $[0, t]$ gives

$$
\begin{aligned}
& e^{\lambda t}\left|u(t)-u_{\infty}\right|^{2} \\
& \leq\left|u(0)-u_{\infty}\right|^{2} \\
& \quad+\left[\lambda+\lambda_{1}\left(-2 \nu+\frac{2 c_{1}}{\sqrt{\lambda_{1}}}\left\|u_{\infty}\right\|+\frac{2 L_{g}}{\lambda_{1}}\right)\right] \int_{0}^{t} e^{\lambda s}\left\|u_{s}-u_{\infty}\right\|_{C_{H}}^{2} d s
\end{aligned}
$$

and consequently,

$$
\begin{aligned}
& \left|u(t)-u_{\infty}\right|^{2} \\
& \quad \leq e^{-\lambda t}\left|u(0)-u_{\infty}\right|^{2} \\
& \quad+\left[\lambda+\lambda_{1}\left(-2 \nu+\frac{2 c_{1}}{\sqrt{\lambda_{1}}}\left\|u_{\infty}\right\|+\frac{2 L_{g}}{\lambda_{1}}\right)\right] \int_{0}^{t} e^{-\lambda(t-s)}\left\|u_{s}-u_{\infty}\right\|_{C_{H}}^{2} d s .
\end{aligned}
$$

As it is straightforward to see that

$$
\left|u(t)-u_{\infty}\right|^{2} \leq e^{-\lambda t} \sup _{\theta \in[-h, 0]}\left|u(\theta)-u_{\infty}\right|^{2} \text { for } t \in[-h, 0],
$$

and

$$
\lambda>\lambda+\lambda_{1}\left(-2 \nu+\frac{2 c_{1}}{\sqrt{\lambda_{1}}}\left\|u_{\infty}\right\|+\frac{2 L_{g}}{\lambda_{1}}\right),
$$

then the exponential stability of $u_{\infty}$ follows from Lemma 8 .

REMARK 10. The above result can also be extended to cover the case in which the operator $g(\cdot, \cdot)$ is defined from $C_{H}$ into $V^{\prime}$ (which is the situation considered in Chen [6] in the case of just one variable delays) but assuming that

$$
\|g(t, \phi)-g(t, \psi)\|_{*} \leq L_{g}\|\phi-\psi\|_{C_{H}}, \text { for all } \phi, \psi \in C_{H} .
$$

Then, the result holds by assuming

$$
\nu>\frac{c_{1}}{\sqrt{\lambda_{1}}}\left\|u_{\infty}\right\|+L_{g}
$$

instead of (33).

Acknowledgements. We would like to thank the referees for their detailed reports and suggestions which allowed us to improve the presentation of this paper. 


\section{References}

[1] A. Bensoussan, G. Da Prato, M.C. Delfour, S.K. Mitter, Representation and Control of Infinite Dimensional Systems, Vol. I, Birkhäuser, Boston-Basel-Berlin, (1992).

[2] T. Caraballo, X. Han, Navier-Stokes models with delays: existence, uniqueness and asymptotic behavior of solutions, in Proceedings of COPDE, London Mathematical Society Lecture Note Series, (to appear) 2014.

[3] T. Caraballo, J. Real, Navier-Stokes equations with delays, R. Soc. Lond. Proc. Ser. A Math. Phys. Eng. Sci. 457 (2001), no. 2014, 2441-2453.

[4] T. Caraballo, J. Real, Asymptotic behaviour of Navier-Stokes equations with delays, R. Soc. Lond. Proc. Ser. A Math. Phys. Eng. Sci. 459 (2003), no. 2040, 3181-3194.

[5] T. Caraballo, J. Real, Attractors for 2D-Navier-Stokes models with delays, J. Differential Equations 205 (2004), no. 2, 271-297.

[6] H. Chen, Asymptotic behavior of stochastic two-dimensional Navier-Stokes equations with delays, Proc. Indian Acad. Sci. (Math. Sci.) 122 (2012), no. 2, 283-295.

[7] P. Constantin, C. Foias, Navier Stokes Equations, The University of Chicago Press, Chicago, (1988).

[8] J. García-Luengo, P. Marín-Rubio, J. Real, Pullback attractors for 2D Navier-Stokes equations with delays and their regularity, Adv. Nonlinear Stud. 13 (2013), no. 2, 331-357.

[9] M.J. Garrido-Atienza, P. Marín-Rubio, Navier-Stokes equations with delays on unbounded domains, Nonlinear Analysis TMA 64 (2006), 1100-1118.

[10] O.A. Ladyzhenskaya, Attractors for Semigroups and Evolution Equations, Cambridge, Cambridge University Press, (1991).

[11] J.L. Lions, Quelques méthodes de résolutions des probèmes aux limites non linéaires, Paris; Dunod, Gauthier-Villars, (1969).

[12] G. Planas, E. Hernández, Asymptotic behaviour of two-dimensional time-delayed NavierStokes equations, Discrete Continuous Dynamical Systems 21 (2008), no. 4, 1245-1258.

[13] T. Taniguchi, The exponential behavior of Navier-Stokes equations with time delay external force, Discrete Continuous Dynamical Systems 15 (2005), no. 5, 997-1018.

[14] R. Temam, Navier-Stokes equations, Theory and Numerical Analysis, 2nd. ed., North Holland, Amsterdam, (1979).

[15] R. Temam, Infinite Dimensional Dynamical Systems in Mechanics and Physics, SpringerVerlag, New York, (1988).

Depto. de Ecuaciones Diferenciales y Análisis Numérico, Facultad de Matemáticas,

Universidad de Sevilla, Campus Reina Mercedes, 41012-Sevilla, SPAIN

E-mail address: caraball@us.es

221 Parker Hall, Department of Mathematics and Statistics, Auburn University, Auburn, AL 36849 USA

E-mail address: xzh0003@auburn.edu 